Adult vaccination as the cornerstone of successful ageing: The case of herpes zoster vaccination. A European Interdisciplinary Council on Ageing (EICA) expert

Formattato: Italiano (Italia)

\title{
focus group
}

Fiona Ecarnot ${ }^{1,2}$, Roberto Bernabei ${ }^{3}$, Giovanni Gabutti ${ }^{4}$, Sandro Giuffrida ${ }^{5}$, Jean-Pierre Michel $^{6}$, Giovanni Rezza7 ${ }^{7}$ Stefania Maggi ${ }^{8}$.

1. Department of Cardiology, University Hospital, Besancon, France

2. EA3920, University of Burgundy Franche-Comté, Besancon, France

3. Dipartimento Scienze dell' Invecchiamento, Neurologiche, Ortopediche e della Testa-Collo, Fondazione Policlinico A. Gemelli, Rome, Italy

4. Department of Medical Sciences, University of Ferrara, Ferrara, Italy

5. Department of Prevention, Local Health Unit Reggio Calabria, Italy

6. University of Geneva, Geneva, Switzerland

7. Department of Infectious Diseases, Istituto Superiore di Sanità, Roma, Italy

8. CNR Aging Branch-NI, Padova, Italy

Address for correspondence:

Fiona Ecarnot

Department of Cardiology

University Hospital Jean Minjoz

3 Boulevard Fleming

25000 Besancon

France

Tel: +33.381.668.539; Fax: +33.381.668.582; Email: Fiona.ecarnot@univ-fcomte.fr Word count: 3515 


\section{Abstract}

Herpes zoster $(\mathrm{HZ})$ is a painful cutaneous rash with vesicular lesions, lasting up to 3 weeks, and caused by reactivation of the latent Varicella Zoster Virus (VZV). It may be associated with complications, the most feared being post-herpetic neuralgia. Effective vaccines are available to prevent $\mathrm{HZ}$, but uptake remains low. We report here the conclusions of an expert Focus Group convened by the European Interdisciplinary Council on Ageing (EICA). The group discussed how existing recommendations regarding $\mathrm{HZ}$ vaccination could be better implemented, and how compliance and coverage with $\mathrm{HZ}$ vaccination could be enhanced. This report proposes strategies to increase awareness of $\mathrm{HZ}$ and its vaccine, enhance vaccine uptake, and educate regarding the role of prevention, including immunization, as a means to "age well". A key strategy that could rapidly and easily be implemented at low cost is co-administration of $\mathrm{HZ}$ vaccine with other vaccines scheduled in the target age group. The scientific evidence surrounding the safety and efficacy of co-administration is discussed. Other strategies, such as active calls, publicity campaigns and national vaccine registries are also outlined. There is a compelling need for a full consensus document that carries weight across all the healthcare professions involved in vaccination, to issue simple and basic recommendations for all healthcare providers. 
Adult vaccinations have been proven to decrease morbidity and mortality linked to preventable infectious diseases [1], reducing complications and hospitalisation in the intensive care unit [2]. Wider use of vaccination is also an effective strategy to decrease antibiotic use and should be the first line of defence against antimicrobial-resistant pathogens [3, 4]. Achieving high vaccine coverage rates not only reduces the incidence of the disease, but also contains the spread of the disease through herd protection, and in the longer term, is also a major contributor to successful ageing, together with healthy lifestyles (non-smoking, good nutrition such as a Mediterranean diet, physical exercise, mental fitness, regular check-ups, good hygiene and midlife disease prevention).

Herpes zoster $(\mathrm{HZ})$ is a painful cutaneous rash with vesicular lesions, which may last between 10 days and 3 weeks, and is caused by reactivation of the latent Varicella Zoster Virus (VZV). Primary infection with VZV occurs in the form of chickenpox, a childhood disease contracted by more than $90 \%$ of children in temperate regions by the age of 10 to 12 years, in the absence of a varicella vaccination programme. After primary infection, the virus remains dormant, primarily in the sensory ganglia, and may be reactivated later in life as herpes zoster, often coinciding with a decline in the individual's immunity, such as the age-related decline known as immunosenescence. This makes old age a powerful risk factor for herpes zoster and older patients may be affected to a greater extent by treatment-related adverse events [5]. Complications of herpes zoster infection can also occur, including post-herpetic neuralgia, defined as pain persisting for more than 90 days after onset of the rash [6]. Effective vaccines are available to prevent $\mathrm{HZ}$, and the literature about $\mathrm{HZ}$ vaccination in older individuals has demonstrated a substantial positive impact in improving quality of life, and in decreasing the complications associated with $\mathrm{HZ}$ infection, including the worsening of co-morbidities. Cost-effectiveness analyses of $\mathrm{HZ}$ vaccination are also very convincing and demonstrated in different settings and target populations [7]. 
Against this background, a group of experts from Italy came together in a Focus Group convened by the European Interdisciplinary Council on Ageing (EICA) to discuss how existing recommendations regarding $\mathrm{HZ}$ vaccination could be better implemented, and how compliance and coverage with $\mathrm{HZ}$ vaccination could be enhanced. Furthermore, the group discussed the strategies that could be implemented to increase awareness of $\mathrm{HZ}$ and its vaccine, and to educate regarding the role of prevention, including immunization, as a means to "age well".

\section{Adult vaccination and vaccine co-administration}

Effective vaccines currently exist against herpes zoster. Live zoster vaccine (ZVL) contains a live attenuated strain of VZV, and has been licensed for use in the USA and Europe since 2006 for the prevention of herpes zoster in adults aged 50 years and older. There is a large body of evidence in the literature attesting to the efficacy of ZVL, including in frail nursing home residents aged 80 years and older [8,9]. A recent retrospective, matched cohort study in 295,135 individuals aged 70 to 79 years from England reported a vaccine effectiveness for preventing shingles of $65.3 \%$ (95\% confidence interval $(\mathrm{Cl}): 60.3-69.6 \%)$ [10]. Epidemiological monitoring since the introduction in England in 2013 of a herpes zoster vaccination programme for adults aged 70 years with a phased catch-up programme for those aged 71-79 years, shows that across the first 3 years of vaccination for the routine cohorts, incidence of herpes zoster fell by $35 \%$, while the incidence of postherpetic neuralgia fell by $50 \%$ [11]. In this study, uptake ranged between $58 \%$ for the recently targeted cohorts and $72 \%$ for the first routine cohort.

Recently, a non-live recombinant subunit vaccine (recombinant zoster vaccine, $R Z V$ ) has been developed. It is given on a two dose schedule (2 to 6 months apart), and contains an 
adjuvant designed to enhance immunogenicity. It demonstrated outstanding efficacy against herpes zoster infection in randomized trials $[12,13]$, with efficacy approaching $90 \%$. Pooled analysis of data from subjects aged 70 years or older from both trials found vaccine efficacy against herpes zoster of $91.3 \%(95 \% \mathrm{Cl}, 86.8$ to $94.5 ; \mathrm{p}<0.001)$ [13]. The protective effective of $R Z V$ is maintained up to 9 years after the initial vaccination, according to the latest data [14].

In Italy, the current vaccine schedule recommends zoster vaccination for all adults aged 65 years and over with ZVL, the only commercially available vaccine. However, knowledge of this recommendation is low, both among the general public and among healthcare providers, particularly general practitioners (GPs). As a result, coverage rates remain low, and substantially lower than target rates. In this regard, the Italian national vaccine plan for the period $2017-2019$ had set a goal of increasing coverage from $20 \%$ in 2017 , to $35 \%$ in 2018 and $50 \%$ in 2019. Currently, it would appear that the reality is lagging behind the target rates, and the $50 \%$ threshold is unlikely to be met in 2019 without drastic measures to improve uptake rapidly. A survey performed by DoxaPharma via 201 individual interviews with patients suffering from post-herpetic neuralgia in Italy found that on average, $74 \%$ of respondents were unaware that free vaccination against herpes zoster was available for subjects over 65 years of age (range from $63 \%$ to over $90 \%$ in some regions of Italy) [15]. The majority also said that they would have gotten the vaccine if they had known about it, and after having had herpes zoster, they would now recommend the vaccine to their friends and/or relatives. Similarly, in a survey of 1001 residents of Ferrara, Italy aged over 50 years, who completed a questionnaire at the local health authority, GP or public health department outpatient clinics, $91 \%$ declared that they were unaware of the herpes zoster vaccine [16]. Acceptability of the vaccine was found to be significantly affected by factors such as age, knowing someone who had suffered from herpes zoster, a 
generally favourable attitude towards vaccination, receiving advice to get the vaccine from their GP, willingness to get vaccinated even on a fee-paying basis [16]. This study suggests some interesting avenues that could be targeted for attempts to raise vaccine awareness and uptake.

The main question then is how can we increase coverage? There are several strategies that can be employed to help improve uptake, and first among these is increasing awareness among the public about the impact of the disease, and the existence of an effective vaccine to prevent it. Publicity campaigns, via television advertisements, traditional posters in doctors' waiting rooms etc, can be used to increase awareness among the general public. Implementing an active call strategy, specifically contacting target age groups by letter or by phone informing them about the vaccine and/or its free availability, has been shown to be highly effective in prompting people to seek information from their healthcare provider, and in many cases, also to obtain the vaccine at the same time. In this regard, improving knowledge of the vaccine among general practitioners (GPs) and pharmacists is also essential, because older people who receive a letter informing them about the vaccine are most likely to turn to their nearest healthcare provider for further information, and this is usually the GP or the community pharmacists. It is essential that these healthcare providers be able to inform the person about the vaccine, including when and where it can be administered to them, so that the opportunity for vaccination is not lost. Pharmacists and GPs have an important role to play as key facilitators of dialogue and information, and they need to receive adequate training and information to ensure that they are competent to fulfil this role.

A national vaccine registry is an extremely useful means of obtaining reliable and up-todate information about coverage, year on year. Robust data is essential to identify gaps in 
coverage, such as geographical areas or specific age-groups that are under-targeted. These data can help to shape public health campaigns and document progress in enhancing uptake in real-world practice.

In the Italian setting, it could be helpful to capitalize on the opportunity offered by the two other vaccines that, together with herpes zoster vaccine, are actively recommended and provided free of charge for the same age group (i.e. over 65s). These are the seasonal influenza vaccine, and the pneumococcal vaccine. Through a phenomenon termed the “carry-over" effect [17], a mandate for administration of one vaccine can increase people's propensity to get other vaccines as well. A US study based on administrative claims data from 31 million Medicare fee-for-service beneficiaries, eligible to receive vaccinations found that claims for pneumococcal, herpes zoster, and tetanus-containing vaccines were mainly concentrated in the same period as the free influenza vaccines, underlining the potential of mandatory appointments as an additional opportunity to assess, recommend, and administer other recommended vaccines [18]. Indeed, vaccine recipients can easily be discouraged by a lack of information, or the perspective of hard-to-reach vaccination centres, waiting lists and additional appointments. In this way, Italian elders who consult for the flu or pneumococcal vaccine are more likely to be open to receiving the herpes zoster vaccine as well, and therefore proposing co-administration of the vaccines is an effective method to take advantage of the patient's presence and willingness to be vaccinated, as well as a relatively inexpensive strategy to achieving higher coverage rates for herpes zoster vaccination by "piggy-backing" on existing healthcare pathways in the older adult population.

\section{2- Safety of vaccine co-administration}


The co-administration of herpes zoster vaccine along with other vaccines recommended in the same target group can only be envisaged if it is safe, well tolerated, and as long as the efficacy of both (or all) co-administered vaccines is not in any way impaired by the concomitant administration. In this regard, there is a large body of evidence attesting to the safety of co-administering ZVL with the influenza vaccine. In a randomized, blinded, placebo-controlled study across 20 sites in Europe and the USA, Kerzner et al investigated the safety and immunogenicity of ZVL administered either concomitantly with inactivated influenza vaccine ( $n=382)$, or sequentially $(n=380)$, in adults aged 50 and older [19]. In terms of the primary safety endpoint, they observed no serious adverse events related to ZVL during the study. In terms of the immunogenicity endpoints, they found that antibody responses to both vaccines were adequate, regardless of whether the vaccines were administered together or sequentially [19]. More recently, in a similar design, Levin et al investigated the safety and immunogenicity of ZVL administration together with quadrivalent inactivated influenza vaccine (IIV4) in adults aged 50 years and older [20]. Again, they also found that the immunogenicity (as assessed by antibody geometric mean titers) after concomitant administration was comparable to that observed after each vaccine alone, and the vaccines were well-tolerated. Overall, current evidence supports the safety and immunogenicity of concomitant administration of influenza and herpes zoster vaccination.

The safety and efficacy of co-administration of herpes zoster vaccine with pneumococcal vaccines has been the subject of some debate. In 2010, Maclntyre et al published the results of a randomized, double-blind, placebo-controlled study that evaluated the safety and immunogenicity of ZVL administered concomitantly versus non-concomitantly with the 23-valent pneumococcal polysaccharide vaccine (PPV23) in 473 subjects [21]. Using an endpoint of antibody response as assessed by enzyme-linked immunosorbent assay 
(ELISA), the authors reported that the antibody geometric mean titer response induced by ZVL administered concomitantly with PPV23 was inferior to that induced by sequential administration, and therefore, the two vaccines should not be administered simultaneously in order to avoid a potential decrease in ZVL immunogenicity [21]. On the basis of these findings, the product labelling recommends an interval of at least 4 weeks between the two vaccines, although the US Advisory Committee on Immunization Practices continues to recommend concomitant administration of these two vaccines [22]. Since then, a large body of real-world evidence using clinical endpoints (namely incidence of both diseases) has accumulated, indicating that concomitant administration of both vaccines provides satisfactory protection. In a first observational study from Kaiser Permanente Southern California, the incidence of herpes zoster after vaccination in the population receiving both herpes zoster and pneumococcal vaccines on the same day was compared to that in the population receiving a pneumococcal vaccine within one year to 30 days prior to the zoster vaccine [23]. After an average follow-up of 1.72 and 1.79 years respectively among the concomitant and non-concomitant cohorts, the authors found no evidence of an increased risk of herpes zoster in the population receiving zoster vaccine and pneumococcal vaccine simultaneously. In an extension of this cohort study, Bruxvoort et al recently reported the results after 9.5 years of follow-up, confirming the previous finding that there is no evidence of vaccine interference [24]. Importantly, this data is based on hard endpoints, namely actual incidence of disease in a real-world population, and not merely an intermediate endpoint of antibody response within a few weeks after vaccine receipt. This is fundamental, as it is has been reported that VZV ELISA antibody titers are not an excellent marker of actual risk of herpes zoster infection [25]. Therefore, relying on antibody-response-assessed immunogenicity to develop recommendations may lead to erroneous interpretations, and substantial lost opportunities for vaccination. 
Regional experiences in Italy provide further evidence to support co-administration of herpes zoster and 13-valent pneumococcal conjugate vaccine at national level. In the Italian region of Calabria, the local health services sought to address the inconvenience barrier by scheduling herpes zoster vaccination at the same time as planned pneumococcal vaccination. They sent detailed invitation letters (the so-called "active call" strategy) to the cohorts concerned, describing the vaccines and underlining the opportunity to receive both vaccinations at the same time. Finally, they also educated the healthcare providers to deliver detailed information regarding the vaccinations during a prevaccination counselling session. Between February 2016 and December 2017, 7490 invitation letters were sent to individuals aged 65 years, and 6179 letters to individuals aged 70 years. Of these, those from the 65 year old cohort who received $\mathrm{HZ}$ vaccine $(n=1693)$ also received PCV13 $(n=1617)$ in $95.5 \%$ of cases, while the co-administration rate was $94.4 \%$ in the 70 year old cohort (HZ vaccine, $n=1281$ and PCV13, $n=1209)$. No vaccine-related adverse events were observed. Such high coverage rates for both vaccines have never previously been achieved in Calabria, highlighting the efficacy of the co-administration strategy in boosting uptake rates. This experience also shows the powerful impact of a simple communication tool, namely active contact via a letter sent to target groups. This inexpensive, yet far-reaching strategy is a strong motivator to prompt target groups into taking the first step onto the vaccination pathway.

\section{Critical issues and obstacles to improved uptake}

A major issue hampering uptake of herpes zoster vaccination is the lack of awareness among the public of the disease. In the Doxapharma survey mentioned earlier [15], around half the respondents were unaware of the incidence of zoster and post-herpetic neuralgia, and of the risk it represents. Naturally, when people don't know about a disease, they don't 
seek vaccination against it. Raising awareness is critical to stimulating demand for the vaccine, particularly since the consequences, namely post-herpetic neuralgia can be especially debilitating. This need for increased knowledge is valid not only for the target population eligible for the vaccine, but also for the GPs and pharmacists, who will be the first line providers of the vaccine. Indeed, the need for cold storage of the vaccine means that many pharmacies and GP offices do not store herpes zoster vaccine on site, and therefore, it is essential that they are able to provide specific and accurate information to patients about when and where to get immunized.

If a large-scale publicity campaign about the disease is implemented on a local, regional or national scale, and if the desired result is achieved (i.e. increased demand), then it is equally important to ensure that the vaccine supply keeps pace, and that vaccine administration centers have sufficient capacity to deliver in the event of a peak in demand. Indeed, vaccine manufacturing processes are slow, and upscaling production in case of increased demand may only result in increased vaccine supply after a period of several months to one year. Therefore, any public health programs intending to stimulate an upsurge in demand should be discussed between the health authorities and the pharmaceutical industry to ensure that the supply will be able to meet the demand. In addition, the recently developed RZV has been approved by the European Medicines Agency (EMA) for marketing in the countries of the European Union, but it is not yet commercially available in Italy. Any public health campaigns targeting the public should be carefully designed, with clear, designated procedures for patients to follow to obtain available vaccines. This is to avoid generating demand for a product that cannot yet be provided.

As seen above, the "active call" strategy is effective in prompting target populations to take action. In the case of wide-scale implementation of such a policy, there are several ethical 
and organizational issues that need to be addressed in advance. The target group must be identified on the basis of the best available scientific evidence, and also according to available organizational capacity. Depending on the size of the target population, it may or may not be feasible to contact wide age ranges; it may be necessary to restrict a first wave of active calls to a single birth cohort, or a smaller age range. Once contacted, as mentioned above, it is essential to ensure that the delivery capacity on the ground is available and functional, to ensure that no opportunities to vaccinate are lost. Once successive waves of active calls have been implemented, the sustainability of the programs and standardization of practices are necessary to ensure that it becomes routine practice implemented in a uniform manner throughout the country. Again, as mentioned earlier, vaccine registries are helpful in this regard, for documenting practice, and providing quality indicators that can be compared across regions and from year to year. A further advantage of vaccine registries, or a similar system for recording the vaccines received by a person over their lifetime, is to follow the timing of vaccine administrations. Indeed, the idea of using scheduled vaccine appointments to promote concomitant administration of a second vaccine needs to be reconciled with the different time schedules for receipt of each type of vaccine. Seasonal influenza vaccination is recommended every year, whereas pneumococcal and herpes zoster vaccines provide protection for several years, and may simply require administration of a booster several years later. There is also evidence that herpes zoster infection can increase the subsequent risk of vascular disease including transient ischemic attack and stroke in a phenomenon known as "VZV vasculopathy" [2630]. Therefore, preventing herpes zoster infection with vaccination would directly reduce the risk of these potentially life-threatening complications. In this regard, an easily accessible record of the person's vaccine history could make it easier to schedule the right vaccines (or boosters) at the right time, to ensure continued protection, especially when older age and waning immunity places the person at highest risk of infection. 
Finally, implementation and sustainability as well as availability are all largely dependent on financing. Strong political will is necessary to ensure that the public health system steps up to the plate and provides the necessary reimbursement for the vaccine, as well as funding for prevention and public information campaigns. There is likely to be some discussion about whether the vaccine should be reimbursed for everyone, or only for target groups and/or patients at high risk. Finding a valid economic model that adequately rewards the healthcare provider for administering the vaccine, and equal reward across healthcare settings (GP, pharmacy, community nurse...) is instrumental in ensuring that the healthcare providers also have a vested interest in striving for high vaccine uptake.

\section{$\underline{\text { Conclusion }}$}

In conclusion, ZVL is safe and effective for the prevention of herpes zoster, and its most debilitating complication, post-herpetic neuralgia. The vaccine has also been shown to be safe and adequately immunogenic when administered concomitantly with other vaccines such as influenza and pneumococcal vaccines. Concomitant administration is a useful strategy to increase coverage, through several mechanisms. Firstly, through a "carry-over" effect, whereby people who are compliant with one vaccination will be more easily convinced to adhere to others, and may be prevailed upon to accept more than one vaccine at scheduled appointments. Secondly, this eliminates the barrier represented by the inconvenience of scheduling additional appointments, or lack of time for multiple consultations, by capitalizing on the opportunities where the patient is already present. Local health authorities, GPs and pharmacists should present a united front and a coherent message in favor of vaccine co-administration, by giving herpes zoster vaccine at the same time as influenza or pneumococcal vaccines. This could be achieved by continuing medical education or training for healthcare providers in the context of a national immunization plan. In this regard, there is a compelling need for a consensus 
document from a national organization that carries weight across all the healthcare professions, such as a national health institute, to issue simple and basic recommendations for all healthcare providers regarding the national policy on immunization. 
References

1. Orenstein WA, Ahmed R: Simply put: Vaccination saves lives. Proc Natl Acad Sci U S A 2017, 114(16):4031-4033.

2. Arriola C, Garg S, Anderson EJ, Ryan PA, George A, Zansky SM, Bennett N, Reingold A, Bargsten M, Miller L et al: Influenza Vaccination Modifies Disease Severity Among Community-dwelling Adults Hospitalized With Influenza. Clin Infect Dis 2017, 65(8):1289-1297.

3. Ginsburg AS, Klugman KP: Vaccination to reduce antimicrobial resistance. Lancet Glob Health 2017, 5(12):e1176-e1177.

4. Howitz MF, Harboe ZB, Ingels H, Valentiner-Branth P, Molbak K, Djurhuus BD: A nationwide study on the impact of pneumococcal conjugate vaccination on antibiotic use and ventilation tube insertion in Denmark 2000-2014. Vaccine 2017, 35(43):5858-5863.

5. Zorzoli E, Pica F, Masetti G, Franco E, Volpi A, Gabutti G: Herpes zoster in frail elderly patients: prevalence, impact, management, and preventive strategies. Aging Clin Exp Res 2018, 30(7):693-702.

6. Schmader K, Gnann JW, Jr., Watson CP: The epidemiological, clinical, and pathological rationale for the herpes zoster vaccine. J Infect Dis 2008, 197 Suppl 2:S207-215.

7. Chiyaka ET, Nghiem VT, Zhang L, Deshpande A, Mullen PD, Le P: CostEffectiveness of Herpes Zoster Vaccination: A Systematic Review. Pharmacoeconomics 2018.

8. Lelic A, Verschoor CP, Lau VW, Parsons R, Evelegh C, Bowdish DM, Bramson JL, Loeb MB: Immunogenicity of Varicella Vaccine and Immunologic Predictors of 
Response in a Cohort of Elderly Nursing Home Residents. J Infect Dis 2016, 214(12):1905-1910.

9. Oxman MN, Levin MJ, Johnson GR, Schmader KE, Straus SE, Gelb LD, Arbeit RD, Simberkoff MS, Gershon AA, Davis LE et al: A vaccine to prevent herpes zoster and postherpetic neuralgia in older adults. N Engl $J$ Med 2005, 352(22):22712284.

10. Matthews I, Lu X, Dawson H, Bricout H, O'Hanlon H, Yu E, Nozad B: Assessing the effectiveness of zoster vaccine live: A retrospective cohort study using primary care data in the United Kingdom. Vaccine 2018.

11. Amirthalingam G, Andrews N, Keel P, Mullett D, Correa A, de Lusignan S, Ramsay $M$ : Evaluation of the effect of the herpes zoster vaccination programme 3 years after its introduction in England: a population-based study. Lancet Public Health 2018, 3(2):e82-e90.

12. Lal H, Cunningham AL, Godeaux O, Chlibek R, Diez-Domingo J, Hwang SJ, Levin MJ, McElhaney JE, Poder A, Puig-Barbera J et al: Efficacy of an adjuvanted herpes zoster subunit vaccine in older adults. N Engl J Med 2015, 372(22):2087-2096.

13. Cunningham AL, Lal H, Kovac M, Chlibek R, Hwang SJ, Diez-Domingo J, Godeaux O, Levin MJ, McElhaney JE, Puig-Barbera J et al: Efficacy of the Herpes Zoster Subunit Vaccine in Adults 70 Years of Age or Older. N Engl J Med 2016, 375(11):1019-1032.

14. Schwarz TF, Volpe S, Catteau G, Chlibek R, David MP, Richardus JH, Lal H, Oostvogels L, Pauksens K, Ravault S et al: Persistence of immune response to an adjuvanted varicella-zoster virus subunit vaccine for up to year nine in older adults. Hum Vaccin Immunother 2018, 14(6):1370-1377. 
15. ["Neuropathic pain from herpes zoster: Patients' experience" Survey]. Available at:

https://www.notiziariochimicofarmaceutico.it/2017/10/23/indagine-il-doloreneuropatico-da-fuoco-di-santantonio/ [Access date: 14 December 2018]

16. Valente N, Lupi S, Stefanati A, Cova M, Sulcaj N, Piccinni L, GPs Study Group, Gabutti G: Evaluation of the acceptability of a vaccine against herpes zoster in the over $\mathbf{5 0}$ years old: an Italian observational study. BMJ Open 2016 , 6(10):e011539.

17. Dempsey AF, Schaffer SE: Human papillomavirus vaccination rates and state mandates for tetanus-containing vaccines. Prev Med 2011, 52(3-4):268-269.

18. Shen AK, Warnock R, Chu S, Kelman JA: Receipt of other routinely recommended vaccines relative to receipt of seasonal influenza vaccines: Trends from medicare administrative data, 2013-2015. Vaccine 2018, 36(30):4399-4403.

19. Kerzner B, Murray AV, Cheng E, Ifle R, Harvey PR, Tomlinson M, Barben JL, Rarrick K, Stek JE, Chung MO et al: Safety and immunogenicity profile of the concomitant administration of ZOSTAVAX and inactivated influenza vaccine in adults aged 50 and older. J Am Geriatr Soc 2007, 55(10):1499-1507.

20. Levin MJ, Buchwald UK, Gardner J, Martin J, Stek JE, Brown E, Popmihajlov Z: Immunogenicity and safety of zoster vaccine live administered with quadrivalent influenza virus vaccine. Vaccine 2018, 36(1):179-185.

21. MacIntyre CR, Egerton T, McCaughey M, Parrino J, Campbell BV, Su SC, Pagnoni MF, Stek JE, Xu J, Annunziato PW et al: Concomitant administration of zoster and pneumococcal vaccines in adults $>/=60$ years old. Hum Vaccin 2010 , 6(11):894-902. 
22. Harpaz R, Ortega-Sanchez IR, Seward JF, Advisory Committee on Immunization Practices Centers for Disease C, Prevention: Prevention of herpes zoster: recommendations of the Advisory Committee on Immunization Practices (ACIP). MMWR Recomm Rep 2008, 57(RR-5):1-30; quiz CE32-34.

23. Tseng HF, Smith N, Sy LS, Jacobsen SJ: Evaluation of the incidence of herpes zoster after concomitant administration of zoster vaccine and polysaccharide pneumococcal vaccine. Vaccine 2011, 29(20):3628-3632.

24. Bruxvoort K, Sy LS, Luo Y, Tseng HF: Real-World Evidence for Regulatory Decisions: Concomitant Administration of Zoster Vaccine Live and Pneumococcal Polysaccharide Vaccine. Am J Epidemiol 2018, 187(9):18561862.

25. Gilbert PB, Luedtke AR: Statistical Learning Methods to Determine Immune Correlates of Herpes Zoster in Vaccine Efficacy Trials. J Infect Dis 2018, 218(suppl_2):S99-S101.

26. Michel JP: Herpes zoster vaccine: a shot to maintain wellbeing. Aging Clin Exp Res 2015, 27(1):1-3.

27. Langan SM, Minassian C, Smeeth L, Thomas SL: Risk of stroke following herpes zoster: a self-controlled case-series study. Clin Infect Dis 2014, 58(11):14971503.

28. Breuer J, Pacou M, Gauthier A, Brown MM: Herpes zoster as a risk factor for stroke and TIA: a retrospective cohort study in the UK. Neurology 2014, 82(3):206-212.

29. Nagel MA: Varicella zoster virus vasculopathy: clinical features and pathogenesis. J Neurovirol 2014, 20(2):157-163.

30. Nagel MA, Gilden D: Update on varicella zoster virus vasculopathy. Curr Infect Dis Rep 2014, 16(6):407. 


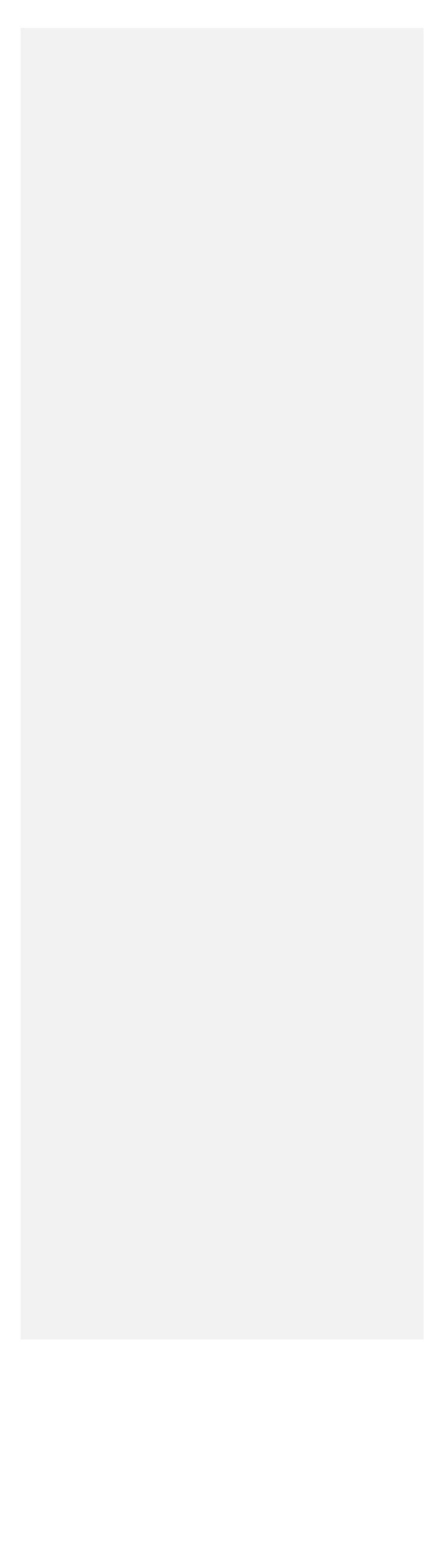

